Hr. Oberbergrath Zinck en zu Mägdesprung, erklärte dasselbe für Sphärosiderit *).

\title{
Botanische Notiz;
}

vom

Professor Dr. Bartling in Göttingen.

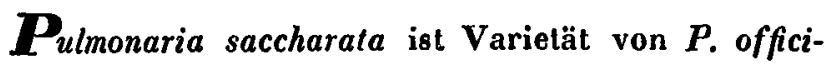
nalis $L$. mit röthlich gefleckten Blättern und kömmt promiscue in vielen Gegenden Deutschlands, z. B. im Donauthale, vor.

Bei einer botanischen Excursion in Pyrmont wurde die hybride Form von Polygonum lapathifolium und $\boldsymbol{P}$. Persicaria häufig in Gräben auf dem Wege nach den Erdfällen angetroffen.

\section{Blutegelzucht, insbesondere die Zuchtteiche in Moritzburg bei Dresden betreffend;} vom Apotheker F, W. Hedrich daselbst.

Die Blutegelzuchtteiche zu Moritzburg wurden in Nurbr. und Decbr. 1834 in der Pressenteichwiese zu achtjährigem Betrieb in $8 \mathrm{Ab}$ theilungen ausgegraben. Jeder Teich ist 20 Ellen lang, 10 breit und 3 tief, der Boden fast durchyängig lehmig mit wenig Sandadern, oben auf $\frac{1}{4}$ Elle guter schwarzer Moor- oder Wiesenboden. Die Teiche sind mit einer Neigung von $45^{\circ}$ ausgegraben, theils weil es für dic Egel bequemer

*) Wahrscheinlich enthielt derselbe dann kohlensaures Eisenoxydul, welches auch im thonigen Sphärosiderit den Hauptbestandthcil ausmacht.

Br. 
ist, theils des Frostes wegen, der sonst durch Heben des Erdreichs die obern trocken liegenden Erdschichten abbröckelt und die Zwischengänge schmälert. Abwärts vom Ufer ist eine, anderthalb Elle in und $\frac{3}{4}$ Elle $\ddot{u} b e r$ die Erde gehende, alle 8 Teiche umschliefsende Bohlenwand angebracht, nach Scheele's Vorschrift, um das Auswandern der Egel zu verhïten, hauptsächlich aber um das Eindringen ihrer Feinde, als des Maulwurfs, des Erdkrebses und der Wasserspitzmaus zu verhindern. Auch sind die Teiche von einem 2 Ellen hohen und 5 Ellen dicken Walle umgeben, bei Wolkenbrüchen und sonst das Ausreifsen der Teiche zu verhüten. Auf diesem Walle steht noch ein $2 \frac{1}{2}$ Elle hoher Zaun mit spanischen Reuterspitzen versehen, um das Einsteigen der unberufenen Liebhaber zu verhindern oder wenigstens zn erschweren. Jeder Teich wurde im Mai 1835 mit 6000 Mutteregeln besetzt, die ich meist in Polen aufkaufte und damals mit 20 Thlr. per 1000 bezahlte. Der Transport in leinenen Beuteln, jeden mit 8-10 Pfd. Egeln angefüllt, ging gut von statten. Man drückt bei dieser Art des Transports die Egel im Beutel gelind aus, um fast alles Wasser zu entfernen, und bindet den Beutel dicht über der Egelmasse zu, so dars der Beutel wie ein angefüllter Geldsack dasteht, und die Egel keinen Raum übrig haben, sich zu bewegen. Die Egel halten sich durch ihre Masse selbst feucht, und werden nur bei warmer Witterung in 2 Tagen, bei mehr kühlem oder Regenwetter in 3 bis 4 Tagen in andere Beutel gebracht, oder wenn sie nicht zu sehr geschleimt haben, nur frisch angefeuchtet. Am Bestimmnngsort angelommen, bringt man die Egel nicht sogleich in die Teiche, sondern schleimt sie erst dadurch ab, dafs man sie über ein Stück aufgespannle Leinwand gleichsam 
hinrollt und dann in ein unterstehendes Gefärs, wie beim Abzählen, fallen lärst. Die Begattungszeit fällt, je nachdem das Frühjahr früher oder später warme Tage bringt, von Mitte Mai bis Mitte Juni, und die ersten Cocons findet man nach dem 10 bis 15 Juli. Die Gröfse der Vermehrung könnte man als die 10fache annehmen, da selten nur 8 , meist 12 bis 14 junge Egel in einem Cocon enthalten sind. Nach dreijährigen, nicht blors einzelnen, sondern mehrfachen Beobachtungen legt ein Blutegel auch mehr als ein Cocon, denn 100 abgesonderte Mutteregel geben mir im Durchschnitt 130 bis 133 Cocons.

Zum Betrieb oder Handel, da die Zuchttèiche so venig wie möglich beunruhigt werden dürfen, ist der sogenannte Entenfang bestimmt, ein schon seit längerer Zeit ausgegrabener Teich von $292 \square$ Ruthen Flächeninhalt, aber nur $1 \frac{1}{4}$ Elle mittlerer Wasserhöhe. In demselben liefs ich im Ferbst 1834, um vor dem Austrocknen und auch Ausfrieren gesichert zu sein, 56 sechs Ellen lange, 4 Ellen breite und 1 Elle tiefere Einschnitte machen, und besetzte ihn nun theils mit 3-4jährigen Egeln, die zum Verkauf noch zu klein waren, theils mit Brut.

Bei dem seit 6-8 Jahren wohl um mehr als den vierfach gestiegenen Werthe der Blutegel war es rathsam, darauf zu merken, dafs die gebrauchten Blutegel nicht mehr als unnütz weggeworfen würden. Gleich: wohl liefsen die früher vorgeschlagenen Mittel, ihnen das Blut durch Ausdrücken zu entziehen, oder sie mit Natron zu bestreuen, die Anwendung nur im Kleinen zu, dürfte auch nicht von Vielen, ohne Ekel zu erregen, befolgt werden. Blors im Nothfall kann man das Mittel gut heifsen. Es wurde daher auf den Vorschlag 
des Hrn. Hof - und Medicinalraths D. Seiler in Dresden, dem wir überhaupt das Entstehen der hiesigen Zuchtteiche, zu verdanken haben, ein besonderer Teich in 2 Abtheilungen, in der sogenannten Grotte im Hirschgarten, mit Canal eingerichtet. Dieser Teich war dazu bestimmt, aufgekaufte Blutegel, welche alle oder doch zum Theil schon gesogen hatten, mindestens ein Jahr lang in demselben ruhen zu lassen, um die Egel so auf natürliche Weise zum Wiedergebrauch geschickt zu machen. Einen Nebennutzen gewähren solche Egel aber noch, wenn sie sonst grofs und alt genug sind, dorch die vermehrte Fortpflanzung. Es ist Schade, dafs ich nur wenig Egel von Wundärzten und Hospitälern zurück erhalte, obgleich ich die Mühe des Sammelns und die tägliche Versorgung derselben mit frischem Wasser, bis ein oder mehre Schock beisammen sind, reichlich genug bezahle.

Wenn nun alle diese Teiche von Sachkennern als ganz zweckmälsig eingerichtet erkannt worden sind, und ich seit dem Herbst 1836 nach der ersten Bekanntmachung mit vielseitigen Aufträgen beehrt wurde, die Anstalt also so zu sagen in gutem Gange ist, so kann ich mich doch keinesweges noch eines Vortheils erfreuen. Nachstehende Schattenseiten will ich für solche nicht verschweigen, die etwa im Begriff sind, eine ähnliche Anstalt, sie sei in kleinem oder grofsem Unfange, zu errichten. Hierbei denke ich an meine Freunde in Dänemark und an die guten örtlichen Gelegenheiten zu einer oder mehrer solcher Anlagen, die in der Gegend von Legeberg oder Bonnhäfft, so wie zwischen Bramstädt und Kellinghusen in Holstein, desgleichen zwischen Eckernförde und Flensburg im Schleswigschen, zu machen wären, ohne gerade andern Orten dortiger Gegend die 
Zulässigkeit solcher Teiche absprechen zu wollen. Ob Dr. Steffens in Christiania, der mir die Noth, in Winter dort Blutegel za erhalten, schilderte, und vor 3 Jahren Willens war, mit Beihülfe der norwegischen Regierung ein solche Anstalt zu errichten, dahin gelangt ist, weirs ich nicht, eben so wenig, ob er die junge Brut von hier aus, die noch in den Cocons eingeschlossen war, damals mit zu Hanse brachte und lebend erhielt. Eine Erwiederung hierauf in dieser weit verbreiteten Zeitschrift dürfte, da die Sache von grofsem practischen Interesse ist, aufser mir auch vielen Andern wilkommen sein.

Die Nachtheile, deren sich meine Blutegelanstalt in neuerer Zeit mehrfach ausgesetzt sieht, sind folgende : Die wilden Enten fallen sowohl am Tage, noch häufiger des Nachts in die Teiche ein. Mit Schiefsgewehr darf man sie hier des Thiergartens wegen nicht verjagen, und sie wegzufangen hält schwer. Die Wasserspitzmaus hat sich seit 2 Jahren trotz der Scheele'schen Vorrichtung eingeschlichen, obgleich noch nicht in Menge. Der Schaden ist aber nicht unbedeutend, da sie gewöhnlich zur Zeit der Coconbildung, wo die Egel tagelang unter dem Rasen verweilen (im Gegensatz zu Scheele's Bemerkung, dem sie sich nur im Winter als schädlich erwiesen), die Lager der Egel anfsuchen, indem sie vom $W$ alle aus ellenhoch über die Bohlenvermachung ins Wasser springen.

Unter die Feinde der Egel oder richtiger der Blategelzuchten mufs ich auch die Diebe zählen. Sie sind die schlimmsten Feinde für meine Anstalten. Da die Teiche in 3 Abtheilungen, jede $\frac{1}{5}$ Stunde von der andern entfernt, liegen, so reicht einWächter unter diesen Umständen nicht ans. So werden denn sowohl bei Tage als bei warmen Nächten die Teiche von ihnen heimgesucht. Die Feinde kommen, wie ich hier leider bemerken mufste, nicht ein- 
zeln, sondern zu 2 oder 3, und wenn ich auch einige derselben der Obrigkeit angezeigt habe, so bleibt mir immer der Schade. Die Diebe hatten sich, um das beschwerliche Uebersteigen zu vermeiden, gerade hinter dem Wachthäuschen zwischen dem Wall und der Vermachung durchgegraben, und treiben so ihr Wesen im Finstern. Uebel ist es, dafs die Egeldiebe ihr Gestohlenes so leicht verwerthen können, und zur Vorsicht, dars ich mein Eigenthum nicht noch einmal bezahle, habe ich grüne (ungarische) und graue (dentsche und polnische) in jeden Teich zusammengethan.

Oben habe ich gesagt, dafs die Vermehrung der Egel durch die Brat als eine 10fache angesehen werden kann. Dies klingt zwar einladend, aber von der Brut verschwindet im ersten Jahre $\frac{4}{5}$ bis $\frac{9}{T 0}$ wieder, wahrscheinlich grofsentheils durch die weifse Erdspinne, die sich häufig an den Ufern solcher Teiche findet. Wäre dieser Umstand nicht, so könnte man erwarten, in der Folge einen reichen Gewinn von solchen Teichen zu ziehen, dessen ich aber vor der Hand noch gänzlich entbehre.

Das Absterben der Egel durch das Aufbewahren derselben im Winter raubt jeden durch den Verkauf im Sommer mit Mühe erlangten Vortheil. 'Hierzn kommt, dafs unsere Herren Collegen im Sommer sich weniger an uns wenden, weil immer noch viele Blutegel von Zuträgern gekauft werden. Der stärkere Vertrieb bleibt mir also für die Wintermonate, daher ich grofse Mengen von Blutegeln aufzubewahren habe. Der Verlust also, den jeder Apotheker durch Absterben der Egel einfach erleidet, trifft mich wohl $50 \mathrm{fach}$, ungeachtet der Anwendung aller vorgeschlagenen Mittel und der Benutzung der eigenen Beobachtungen.

Im Interesse der Apotheker sowohl, als meiner 
Anstalt, suchte ich vor mehren Jahren für Absendung der Blutegel von hier aus an sächsische Apotheker am Portofreiheit nach. Dieser kleine Vortheil ist mir aber nicht gewährt worden. Wer da glaubt, über die angedeuteten Schwierigkeiten hinwegzukommen, gegen Diebstahl und die Wasserspitzmaus gesichert zu sein, wer nicht nöthig hat, den Winter über Vorrath feil zu halten, übrigens guten Wiesenboden besitzt und den nöthigen geregelten Wasserstand herbeizuführen weirs, der mag es immer wagen, eine solche Anlage zu etabliren. Als Leitfaden kann ihm hierbei zur ersten Einrichtung dienen: Scheel, der medic. Blutegel. Breslau 1833, bei Korn; Belehrung und Aufbewahrung des med. Blutegels, vom Hofrath Seiler, Dresden 1834, Walthersche Hofbuchhandlung. Aufserdem sind in diesem Archiv fast in jedem Jahrgange, so wie in Buchn. Repert. als auch dem pharm. Centralbl. eine Menge Notizen über Naturgeschichte, Behandlung und Aufbewahrungsarten und Krankheiten des Blutegels mitgetheilt. Zusammengezogen findet man diese Notizen im Hauslexikon 1. Bd. Leipzig 1834, bei Breitkopf $u$. Härtel. Auch spricht sich Martius im Lehrb. der pharm. Zoologie, Stuttg. 1838, Balz'sche Buchh. ausführlicher darüber aus.

Zum Ausgraben von 8 Zuchtteichen, so wie zur Einfriedigung, wurde mir zu seiner Zeit unter hoher Mitwirkung aus den Staatskassen ein Zuschufs von 600 Thlr. bewilligt. Zum ersten Besetzen der Teiche waren 18321600 Thlr. veranschlagt. Ehe es aber im Herbst 1834 bis zum Abtreten der hierzu bestimmten fiskalischen Grundstücke kam, war der Preis der Egel um das Doppelte gestiegen, so dals ich über 3200 Thlr. zum ersten Ankauf an Egeln aufwenden mufste. Diese zweijährige Zïgerung änderte nichts in der Anlage, wohl aber 
gereichte sie sehr zu meinem Nachtheile wegen der Erhöhung des auf die Egel zu wendenden Capitals.

Wie sehr man schon seit langer Zeit einsah, dafs fïr die immer theurer werdenden Blutegel viel Geld aus dem Lande gehe, und dafs eine Abhülfe wünschenswerth sei, zeigte, aufser der von hoher Staatsregierung auf die oben angeführte Art bewiesenen Begünstigung zur Einrichtung der hiesigen Zuchtteiche, eine öffentliche Bekanntmachung der Königl. sächs. Amtshauptmannschaft zu Chemnitz vom 1. Sept. 1835, wonach aus Privatmilteln der Apotheker D e h ne in Penig sechs, und später acht Louisd'or demjenigen als Prämie aussetzte, der die Blutegelzucht im Kleinen, d. h. jedem Apotheker oder Andern möglich, so weit bringe, dafs nicht nur die von ihnen aus der Brut gezogenen Egel zum medicinischem Gebrauch herangewachsen, sondern auch von der Gröfse seien, dafs sie sich wieder fortpflanzen. Der Termin für die Preisbewerber war der December 1837, auch durfte der Bewerber nicht weiter, als bis zu drei Meilen von Chemnitz heimathsangehörig sein.

Im August 1838 erfolgte die anderweitige amtshanptmannschaftliche Bekanntmachung, dafs dem Rathmann und Wundarzt C. Müller in Penig, welcher die Egel anf zweckmärsige Art in Fässern, bis zum medic. Gebrauch gezogen habe, der Preis von 8 Louisd'or zuerkannt worden sei. Der zweite Punct, selbstgezogene Blutegel bis zur Fortpflanzung zu bringen, scheint aber nicht erledigt zu sein, wenigstens sagt die öffentliche Bekanntmachung nichts davon. Es sei mir daher erlaubt, vor der Hand noch an dieses erreichte Ziel zu zweifeln. Ich habe 5 - 6jährige selbstgezogene Egel, welche zum medicinischen Gebrauch recht gut angehen, aber bis zur Fortpflanzung gehört eine doppelte Grörse und also auch Arch. d. Pharm. II. Reihe. XXII. Bds. 2. Hft. 
ein höheres Alter derselben. Scheel, der wohl die älteste Blutegelzacht besitzt, ist vielleicht der einzige, der es schon so weit gebracht haben kann.

Mit dieser kurzen Schilderung des jetzigen Standes der Blutegelzucht habe ich übrigens Niemanden 20 nahe treten wollen, und indem ich nicht wïnsche, den Anstalten noch mehr Feinde hervorzurufen, bin ich mehr dem eignen Antriebe als der mehrseitigen Aufforderung dazu gefolgt*).

Sechste Abtheilung.

\title{
Toxi k o o g i e.
}

\section{Untersuchung einer Vergiftung durch Schwefelsäure;}

\author{
von \\ L. Aschoff,
}

Apotheker in Bielefeld und Assessor des Vereins.

Unter den zufälligen Vergiftungen, die heut $z u$ Tage leider noch so oft durch Nachlässigkeit herbeigeführt werden, sind wohl die durch Schwefelsäure die häufigsten, wenigstens sind mir seit Kurzem mehre der Art vorgekommen, wovon ich eine ihres auffallenden

*) Obgleich über die immer dringlicher hervortretende Blutegelzucht schon so viel geschrieben worden ist, so scheinen uns doch die Mittheilungen solcher Ergebnisse, wie die vorstehenden, recht practisch-nützlich. Es wäre wünschenswerth, wenn die Besitzer grofser und kleiner Blutegelcolonien über den Ertrag derselben kurze statistische Nachrichten mittheilten. Man würde mit der Zeit dadurch in den Stand gesetzt worden, die entgegenstehenden Schwierigkeites za besiegen und iber den wirklichen Nutzen der Blutegeleucht zu entecheiden.

D. Red. 